\title{
COMPOSITION ALGEBRAS OF DEGREE TWO
}

\author{
by ALBERTO ELDUQUE* and JOSÉ M. PÉREZ-IZQUIERDO*
}

(Received 2nd April 1998)

\begin{abstract}
Composition algebras in which the subalgebra generated by any element has dimension at most two are classified over fields of characteristic $\neq 2,3$. They include, besides the classical unital composition algebras, some closely related algebras and all the composition algebras with invariant quadratic norm.
\end{abstract}

1991 Mathematics subject classification: 17 A75.

\section{Introduction}

An algebra $A$ with product $x y$ over a field $F$ is called a composition algebra if there exists a quadratic form $n(): A \rightarrow F$ such that for any $x, y \in A$

$$
n(x y)=n(x) n(y)
$$

and the bilinear form $n(x, y)=n(x+y)-n(x)-n(y)$ is nondegenerate. Since $n(x, x)=$ $2 n(x)$, over fields of characteristic not 2 we will consider the bilinear form $(x, y)=\frac{1}{2} n(x, y)$ instead of $n($,$) , so n(x)=(x, x)$.

The first well known composition algebras were those with unit element $[13,14]$, also termed Hurwitz algebras. The generalized Hurwitz's Theorem [13, 26] establishes that they are isomorphic to either the base field $F$ (char $F \neq 2$ ), $F \oplus F$, a separable quadratic extension of $F$, a generalized quaternion algebra or a Cayley-Dickson algebra. In particular, the dimension of any Hurwitz algebra is $1,2,4$ or 8 .

Hurwitz algebras are the main source to construct finite dimensional composition algebras without unit element [14]: Given a Hurwitz algebra with product $x \circ y$, quadratic form $n()$ and $\phi, \psi$ two isometries of $n()$, then the new product

$$
x y=\phi(x) \circ \psi(y)
$$

defines a new composition algebra with the same quadratic form $n()$. Contrary to the

\footnotetext{
* Work supported by the Spanish DGICYT (Pb 94-1311-C03-03). The first author also acknowledges support from the "Universidad de La Rioja" (API-97/B05) and the second from grants from the "Plan de Formación del Personal Investigador" and "Programa de Formación del Personal Investigador en el Extranjero", DGICYT.
} 
original algebra, this new algebra is, in general, non-unital. Conversely, given a finite dimensional composition algebra $A$ with product $x y$ and quadratic form $n()$, Kaplansky [14] proved that for any element $a \in A$ with $n(a) \neq 0$ the left and right multiplication operators by $u=a^{2} / n(a)$ (denoted by $L_{u}$ and $R_{u}$ ) are isometries of $n($ ). So the new product

$$
x \circ y=\left(R_{u}^{-1} x\right)\left(L_{u}^{-1} y\right)
$$

gives a composition algebra with attached quadratic form $n()$, and the element $u^{2}$ becomes the unit. As an immediate consequence, any finite dimensional composition algebras has dimension $1,2,4$ or 8 .

In general, over algebraically closed fields, altering the product $x \circ y$ of a Hurwitz algebra $(A, 0)$ as in (2) leads to a new composition algebra with "fewer degrees of symmetry" (i.e. a poorer derivation algebra) except for the following products:

$$
\begin{array}{llll}
\text { (I) } x \circ y & \text { (II) } \bar{x} \circ y & \text { (III) } x \circ \bar{y} & \text { (IV) } \bar{x} \circ \bar{y}
\end{array}
$$

where $x \mapsto \bar{x}$ denotes the standard involution of the Hurwitz algebra $(A, 0)[10]$. The new algebras we obtain in this way are called standard composition algebras (of type $I, \ldots$, IV respectively) associated to the original Hurwitz algebra $(A, 0)$. Standard composition algebras of type IV are also referred as para-Hurwitz algebras [20, 21]. Although all standard composition algebras agree to be the base field $F$ when the dimension of $A$ is one, standard composition algebras of different type are not isomorphic in higher dimensions. With the exception of the two dimensional paraHurwitz algebras, the forms of standard composition algebras are again standard of the same type [10].

The bilinear form of para-Hurwitz algebras satisfies the following equation:

$$
(x y, z)=(x, y z)
$$

i.e. the bilinear form is associative. For any algebra, the composition identity (1) and the previous one are equivalent to the following:

$$
(x y) x=(x, x) y=x(y x)
$$

Moreover, in that case the dimension is necessarily finite [20]. Such algebras are called symmetric [15].

Motivated by work on the SU(3) particle physics, Okubo and Osborn proved that, over algebraically closed fields of characteristic not 2, any symmetric composition algebra is either a para-Hurwitz algebra or a pseudo-octonion algebra [20, 21]. Given an algebraically closed field $F$ (char $F \neq 2,3$ ), the pseudo-octonion algebra $\mathrm{P}_{8}(F)$ over $F$ is defined from the set of 3 by 3 trace zero matrices by changing the usual matrix product $x y$ by 


$$
x * y=\mu x y+(1-\mu) y x-\frac{1}{3} \operatorname{trace}(x y) I_{3}
$$

where $\mu$ is a root of the equation $3 \mu(1-\mu)=1$ and $I_{3}$ is the 3 by 3 identity matrix. The quadratic form $n(x)=\frac{1}{6} \operatorname{trace}\left(x^{2}\right)$ allows composition for the new product $*$. A general definition of pseudo-octonions, valid over any field, can be found in [9]. Given $\bar{F}$ the algebraic closure of $F$, the forms of $\mathrm{P}_{8}(\bar{F})$ are called Okubo algebras. They have been classified by Elduque and Myung in [6] and by Elduque in [3, 4].

In general, to classify all finite dimensional composition algebras is an open problem. However, if we impose certain general identities on these algebras then such a classification can be afforded. Symmetric composition algebras have been studied in [20, 21]. In [19] Okubo shows that, over fields of characteristic not 2, any finite dimensional composition algebra verifying the flexible identity $(x y) x=x(y x)$ is either a form of a Hurwitz algebra, a form of a para-Hurwitz algebra or an Okubo algebra. In [7] Elduque and Myung find, over fields of characteristic not 2 or 3, an equivalence between the category of finite dimensional flexible composition algebras without unit element and the category of separable alternative algebras of degree 3 . This approach leads to the same results as in [19]. In [8] we study finite dimensional composition algebras satisfying the identity $x x^{2}=x^{2} x$ (third power associative law). After long computations we conclude that these algebras do not contribute any new examples because they become either Hurwitz algebras or symmetric algebras.

The study of infinite dimensional composition algebras is much more involved. Although Kaplansky [14] thought those algebras could not exist, Urbanik and Wright [25] gave a whole family of infinite dimensional absolute valued algebras. An absolute valued algebra is an algebra over the real numbers with a norm II such that $|x y|=|x||y|$ for any $x$ and $y$. In case this norm arises from a scalar product (,), i.e. $|x|=\sqrt{(x, x)}$, as it happens to be in the examples given by Urbanik and Wright, we get infinite dimensional composition algebras.

The existence of one sided unity is not enough to force a composition algebra to be finite dimensional $[1,11,23]$. In fact, there are not many conditions under which we can claim that a composition algebra is finite dimensional. An algebra $A$ is called algebraic if the subalgebra generated by any element $x, \operatorname{alg}\langle x\rangle$, is finite dimensional. The degree of $A$ is the supremum of $\{\operatorname{dim} \operatorname{alg}(x)\} \mid x \in A$. The first step in the study of this property for absolute valued algebras has been carried out by Rodríguez Palacios in [24]:

Theorem 1. Any absolute valued algebra of degree 2 is finite dimensional and it is isomorphic to a standard composition algebra or an Okubo algebra.

More recently, it has been proved, by means of topological arguments, that the dimension of any algebraic absolute valued algebra is finite [12].

In this paper we are concerned with an analogue of Theorem 1 for arbitrary composition algebras. The main result is the following:

Main Theorem. Let $A$ be a composition algebra of degree 2 over a field with more 
than five elements and characteristic $\neq 2,3$. Then the dimension of $A$ is finite and $A$ is isomorphic to one of the following algebras: a standard composition algebra, a form of $a$ two dimensional para-Hurwitz algebra or an Okubo algebra.

Using this result, we will give in Section 4 a drastically simpler proof of the classification of third power associative composition algebras [8].

\section{Composition algebras of degree two}

The simpler composition algebras of degree 2 are those of dimension 2 , which have been classified by Petersson [22] (where the extra hypotheses of the characteristic of the ground field to be $\neq 2$ is imposed, but the proof can be adapted to suppress this):

Proposition 1. Let $A$ be a two-dimensional composition algebra with product denoted by $x y$. There can be defined a new product $\circ$ on $A$ such that $(A, 0)$ becomes a Hurwitz algebra and the product $x y$ is given by one of the following formulae:

$$
\begin{array}{llll}
\text { (I) } x \circ y & \text { (II) } \bar{x} \circ y & \text { (III) } x \circ \bar{y} & \text { (IV) } u \circ \bar{x} \circ \bar{y}
\end{array}
$$

where $n(u)=1$ and $x \mapsto \bar{x}$ denotes the standard involution on $(A, \circ)$.

Looking at the algebras in the previous proposition we can check that only those of type I and IV are third power associative algebras [8]:

Proposition 2. Let $A$ be a two-dimensional composition algebra over a field with at least three elements. The following statements are equivalent:

(i) $A$ is commutative.

(ii) $A$ is flexible.

(iii) $A$ is third power associative.

(iv) The product of $A$ is given by formulas (I) or (IV) in the previous proposition.

(v) $A$ is either a Hurwitz algebra or a form of a para-Hurwitz algebra.

Proof. Only (iii) implies (iv) requires some care. Because of the assumptions on the field, the scalars can be extended; so then assume that the ground field is algebraically closed. Then it is straightforward to check that the unique algebra of type II and the unique one of type III are not third power associative.

The result above is not valid over the field of two elements [2].

Note that Proposition 1 together with part (iv) and (v) in the previous proposition says that over algebraically closed fields any composition algebra of dimension 2 is standard. 
Given two standard composition algebras of the same type, it is easy to check that they are isomorphic if and only if their associated Hurwitz algebras are. Since over any algebraically closed field $F$ there exists, up to isomorphism, just one Hurwitz algebra of dimension 2, namely $F \oplus F$, we can conclude that over such fields there are, up to isomorphism, only four composition algebras of dimension 2: the standard composition algebras. A straightforward computation using the identity $x^{2}-n(x, 1) x+n(x) 1=0$ valid in any Hurwitz algebra [26] shows that those algebras can be distinguished from each other by the identities they verify:

\begin{tabular}{|c|c|}
\hline Type & Identities \\
\hline I & $x^{2} x=x x^{2},\left(x^{2}\right)^{2}=\left(x^{2} x\right) x$ \\
\hline II & $x^{2} x=n(x) x,\left(x^{2}\right)^{2}=n(x) x^{2}$ \\
\hline III & $x x^{2}=n(x) x,\left(x^{2}\right)^{2}=n(x) x^{2}$ \\
\hline IV & $x x^{2}=n(x) x=x^{2} x$ \\
\hline
\end{tabular}

Table 1

These pairs of identities will be referred to as identities of type I, .., IV respectively. The goal of this section is to prove that, in fact, any composition algebra of degree 2 satisfies one of the previous pairs of identities. In order to get this it will be useful to work with the Zariski topology (over finite dimensional subspaces), so we will start by extending scalars to an infinite field.

Lemma 1. Let $A$ be an algebra of degree 2 over a field $F$ with more than five elements. For any field extension $K / F$ we have that $A_{K}:=K \otimes_{F} A$ is an algebra of degree 2.

Proof. Denote by $A \wedge A \wedge A$ the subspace of the Grassmann algebra over $A$ spanned by the homogeneous elements of degree 3. Since $F$ contains more than five elements and $A$ is an algebra of degree 2 , then the identities $x \wedge x^{2} \wedge x x^{2}=0=$ $x \wedge x^{2} \wedge x^{2} x=x \wedge x^{2} \wedge\left(x^{2}\right)^{2}$ as well as their linearizations are satisfied. So they remain valid on $A_{K} \wedge A_{K} \wedge A_{K}$, which says that $A_{K}$ is also an algebra of degree 2.

From now on, the characteristic of the ground field will always be assumed to be different from 2.

As we will see below, in any composition algebra of degree 2 the elements generating composition subalgebras of dimension exactly 2 will play a special role. Since the coordinate matrix of the bilinear form on $\operatorname{span}\left(x, x^{2}\right)$ is

$$
\left(\begin{array}{cc}
n(x) & \left(x, x^{2}\right) \\
\left(x, x^{2}\right) & n(x)^{2}
\end{array}\right)
$$

these elements can be characterized by the inequality $\left(x, x^{2}\right)^{2} \neq n(x)^{3}$. In particular, 
the set of these elements lying in any finite dimensional subspace is open in the Zariski topology of that subspace, provided it is nonempty. In [8] many involved computations were necessary to prove that, in general, this set is not empty, that is, we do not have the identity $\left(x, x^{2}\right)^{2}=n(x)^{3}$. The point there was to derive many new identities from this one and to analyze how they fit with the hypothesis of the algebra being third power associative. However, now we can remove this case just by means of the following general lemma:

Lemma 2. Let (, ) be a symmetric bilinear form defined on an algebra of possibly infinite dimension over $a$ field of characteristic $\neq 2$. Consider $B$, a finite dimensional subspace where the bilinear form is nondegenerate. If $\left(x, x^{2}\right)^{2}=n(x)^{3}$ for any $x \in B$ then $\operatorname{dim} B \leq 1$.

Proof. First let us suppose that the field contains more than six elements. Under this assumption we can linearize the identity $\left(x, x^{2}\right)^{2}=n(x)^{3}$ and extend scalars to an infinite field that we can think of as our underlying field. Over this field the algebra of polynomial functions over $B$ is isomorphic to the algebra of polynomials on $m=\operatorname{dim} B$ indeterminates, which it is well known to be a U.F.D. So we can consider how our polynomial $n(x)=(x, x)$ factorizes. Since $n(x)$ is homogeneous of degree 2 then it is either irreducible or it can be written as $n(x)=p_{1}(x) p_{2}(x)$ or $n(x)=p(x)^{2}$ where $p_{1}(x), p_{2}(x)$ and $p(x)$ are irreducible linear polynomials. We can rule out the first two possibilities just by comparing the factorizations that we obtain from the identity $\left(x, x^{2}\right)^{2}=n(x)^{3}$. So we are left with the case $n(x)=p(x)^{2}$. Linearizing this expression we get $(x, y)=p(x) p(y)$, which says that the kernel of the linear form $p(x)$ is contained in the radical of the restriction of $($,$) to B$. Since, by hypothesis, this radical is zero we conclude that $\operatorname{dim} B \leq 1$.

Now suppose the field only contains 3 or 5 elements. In the first case the identity $\left(x, x^{2}\right)^{2}=n(x)^{3}$ ensures that $(x, x)=0,1$ for any $x \in B$; in the latter, it gives $(x, x) \in$ $\{0, \pm 1\}$ for any $x \in B$. If the dimension of $B$ were $\geq 2$ then, in both cases, we could find orthogonal elements $x, y \in B$ such that $(x, x)=1=(y, y)$ so $(x+y, x+y)=2$, which is not possible.

In the following $A$ will denote a composition algebra of degree 2 and dimension $>1$ over an algebraically closed field $F$. Fix $S=\left\{x \in A \mid n(x)^{3}-\left(x, x^{2}\right)^{2} \neq 0\right\}$ and $B$ a finite dimensional subspace of $A$ where the bilinear form is nondegenerate and $\operatorname{dim} B>1$. The previous lemma says that $B^{\prime}=\left\{x \in B \mid n(x)^{3}-\left(x, x^{2}\right)^{2} \neq 0\right\}$ is nonempty, so it is an open set in the Zariski topology of $B$. Because any element in $B^{\prime}$ generates a composition subalgebra of $A$ of dimension two, such an element verifies one type of the identities in Table 1 . So, $B^{\prime}$ can be decomposed as the disjoint union of four closed sets $B_{1}, \ldots, B_{I V}$ where $B_{J}^{\prime}=\left\{x \in B^{\prime} \mid x\right.$ satisfies the identities of type J $\}$. In fact, these sets are also open because any of them is the complement in the open set $B^{\prime}$ of the union of the others. However, in the Zariski topology two nonempty open sets intersect each other, so the only way to fit this situation is that $B^{\prime}=B^{\prime}$, for some $\mathrm{J} \in\{I, \mathrm{II}, \mathrm{III}, \mathrm{IV}\}$, i.e., all elements in $B^{\prime}$ verify the same identities. By density this remains 
true for $B$. Moreover, it is enough to observe that we can extend $B$ to contain any predetermined element of $A$ to conclude that: all the elements in $A$ satisfy the same type of identities, and all the two dimensional composition subalgebras of $A$ have exactly the same type!!

\section{Proof of the main theorem}

Because of the previous section, in order to prove the main theorem we can assume that $A$ satisfies one type of the identities I, .., IV.

The classification of composition algebras where $x x^{2}=x^{2} x$ and $\left(x^{2}\right)^{2}=\left(x^{2} x\right) x$ has been tackled in $[2,18]$ :

Theorem 2. Any composition algebra verifying the identities $x x^{2}=x^{2} x$ and $\left(x^{2}\right)^{2}=\left(x^{2} x\right) x$ is a Hurwitz algebra.

The following theorem deals with the identities of type II and III.

Theorem 3. Let $A$ be a composition algebra of degree 2 and arbitrary dimension over a field $F$ of characteristic $\neq 2$ containing more than 3 elements. Then:

(i) If $x^{2} x=n(x) x$ and $\left(x^{2}\right)^{2}=n(x) x^{2}$ for any $x \in A$, then $A$ is a standard composition algebra of type II.

(ii) If $x x^{2}=n(x) x$ and $\left(x^{2}\right)^{2}=n(x) x^{2}$ for any $x \in A$, then $A$ is a standard composition algebra of type III.

Proof. First of all we should note that it is enough to prove only one part of the theorem because if we have a composition algebra $A$ of degree two, then the opposite algebra $A^{\text {op }}$ is also a composition algebra of degree two, and $A$ is standard of type II (resp. satisfies the identities of type II) if and only if $A^{\text {op }}$ is standard of type III (resp. satisfies the identities of type III). Therefore, let us prove part $i$.

The restrictions on $F$ allow us to linearize the identities in the statement:

$$
\begin{aligned}
x^{2} y+(x y+y x) x & =2(x, y) x+n(x) y \\
(x y+y x) x^{2}+x^{2}(x y+y x) & =n(x)(x y+y x)+2(x, y) x^{2} .
\end{aligned}
$$

Now, take any idempotent $e$ with $n(e)=1\left(x^{2} / n(x)\right.$ where $n(x) \neq 0$ will serve $)$. Choosing $x=e$ and $y$ orthogonal to $e$ in equations (7) we obtain:

$$
\begin{aligned}
e y+(e y+y e) e & =y \\
(e y+y e) e+e(e y+y e) & =e y+y e .
\end{aligned}
$$

The second equation tells us that the operator $L_{e}+R_{e}$ decomposes the orthogonal subspace 
to $e$ in two subspaces associated to the eigenvalues 0 and $1, \operatorname{span}\langle e\rangle^{\perp}=S(0) \oplus S(1)$. For any $x_{0} \in S(0),(8)$ gives:

$$
e x_{0}=x_{0}=-x_{0} e
$$

hence, for any $x_{1} \in S(1)$ we have:

$$
\left(x_{0}, x_{1}\right)=\left\{\begin{array}{l}
\left(e x_{0}, e x_{1}\right)=\left(x_{0}, e x_{1}\right) \\
\left(x_{0} e, x_{1} e\right)=-\left(x_{0}, x_{1} e\right)
\end{array}\right.
$$

which gives $\left(x_{0}, x_{1}\right)=\left(x_{0}, e x_{1}+x_{1} e\right)=0$; that is, $S(0)$ and $S(1)$ are orthogonal. On the other hand, from (7) with $x=x_{1}$ and $y=e$ we obtain:

$$
x_{1} x_{1}^{2}+x_{1}^{2} x_{1}=n\left(x_{1}\right) x_{1} .
$$

Since, by hypothesis, $x_{1}^{2} x_{1}=n\left(x_{1}\right) x_{1}$ it follows that $x_{1} x_{1}^{2}=0$ and $n\left(x_{1}\right)=0$ for any $x_{1} \in S(1)$. So, we conclude that $S(1)$ is the orthogonal complement of $S(0)$ in $\operatorname{span}\langle e\rangle^{\perp}$ and that it is totally isotropic. By the nondegeneracy of $($,$) this means that S(1)=0$ and $A=\operatorname{span}\langle e\rangle \oplus S(0)$. In the light of this, (9) says that $L_{e}$ and $R_{e}$ are bijective and that $A$ with the new product

$$
x \circ y=\left(R_{e}^{-1} x\right)\left(L_{e}^{-1} y\right)=\left(R_{e}^{-1} x\right) y
$$

is a Hurwitz algebra with unit element $e$ and standard involution $x \mapsto \bar{x}=x e$. In particular, $x y=\bar{x} \circ y$ and $A$ is standard of type II.

Theorem 4. Let $A$ be a composition algebra of any, possibly infinite, dimension over a field of characteristic $\neq 2,3$. If $x x^{2}=n(x)=x^{2} x$ for all $x \in A$ then $A$ is symmetric (so, in particular, it is finite dimensional).

Proof. Extending scalars, we can assume the field is infinite. For any $x$ with $n(x) \neq 0$ we have:

$$
(x y, x)=\frac{n(x)}{n(x)}(x y, x)=\frac{1}{n(x)}\left(x y, x x^{2}\right)=\left(y, x^{2}\right),
$$

and, similarly,

$$
(y x, x)=(x y, x)=\left(y, x^{2}\right) .
$$

The set $\{x \in B \mid n(x) \neq 0\}$ is open in the Zariski topology of any not totally isotropic finite dimensional subspace $B$, so the previous identities work not only for elements with nonzero norm, but for any element of $A$. Linearizing these identities we get 


$$
\begin{aligned}
& (x y-z x, y)=(x, z y-y z) \\
& (x z+z x, y)=(x, z y)+(x y, z) .
\end{aligned}
$$

Subtracting these equalities

$$
2(z x, y)=(x y, z)+(x, y z)
$$

We can make a cyclic permutation of $x, y, z$ in the last equation to obtain

$$
2(x y, z)=(y z, x)+(y, z x) .
$$

Adding (10) to twice this equation we have

$$
3(x y, z)=3(y z, x) .
$$

But, since the characteristic of the base field is not 3 , we can divide by 3 and conclude that $A$ is a symmetric composition algebra.

The main theorem is now the consequence of Lemma 1 and Theorems 2, 3 and 4.

\section{Third power associative composition algebras}

The classification of third power associative composition algebras has been carried out in [8]:

Theorem 5. Let $A$ be a finite dimensional composition algebra, over a field of characteristic $\neq 2$ and 3, verifying the identity $x x^{2}=x^{2} x$. Then $A$ is either Hurwitz or symmetric.

As we mentioned in the introduction, the proof we presented in [8] was based on many involved computations that did not throw enough light on what is going on for these particular algebras. So, we would like to devote this section to give a more understandable proof of Theorem 5 based on the previous classification of composition algebras of degree 2 . The point is to prove that any third power associative composition algebra has degree at most 2 (Proposition 3). We will use some results from [8] that we include for completeness.

Hereafter $\boldsymbol{A}$ will denote a finite dimensional composition algebra, over a field $F$ of characteristic not 2 , satisfying the identity

$$
x x^{2}=x^{2} x
$$

which is equivalent to $\left[x, x^{2}\right]=0$, where $[x, y]=x y-y x$ denotes the commutator of $x$ and $y$. The restriction on $F$ allows a scalar extension, so we will assume that $F$ is an 
infinite field. Our starting point is the following lemma [6] that we will apply to $x$ and $x^{2}$.

Lemma 3. Let $A$ be a finite dimensional composition algebra, and $x, y$ two commuting elements of $A$. Then

$$
n(x) y^{2}+n(y) x^{2}=2(x, y) x y
$$

This result also extends to three elements.

Lemma 4. In any finite dimensional composition algebra, any elements $x, y, z$ commuting with each other satisfy

$$
(y, z) x^{2}+n(x) y z-(y, x) x z-(z, x) x y=0 .
$$

If we set $y=x^{2}$ in (12) we obtain

$$
n(x) x^{2} x^{2}+n(x)^{2} x^{2}=2\left(x, x^{2}\right) x^{3} .
$$

The following lemma will be helpful:

Lemma 5. The set $S=\left\{x \in A \mid n(x)\left(x, x^{2}\right)\left[n(x)^{3}-\left(x, x^{2}\right)^{2}\right] \neq 0\right\}$ is dense in the Zariski topology.

Proof. The set $\{x \in A \mid n(x) \neq 0\}$ as well as $\left\{x \in A \mid n(x)^{3}-\left(x, x^{2}\right) \neq 0\right\}$ are open and nonempty (Lemma 2), so they are dense. Since any two open sets in the Zariski topology intersect each other, $S_{1}=\left\{x \in A \mid n(x)\left[n(x)^{3}-\left(x, x^{2}\right)^{2}\right] \neq 0\right\}$ is nonempty, open and dense. Therefore, in order to prove the lemma it is enough to show that $S_{2}=\left\{x \in A \mid\left(x, x^{2}\right) \neq 0\right\}$ is nonempty. If, on the contrary, $\left(x, x^{2}\right)=0$ for any $x$ then take $x \in S_{1}$. By (13) the element $e=-x^{2} / n(x)$ is an idempotent of norm 1, so $\left(e, e^{2}\right)=(e, e)=1$, which contradicts the assumption.

Note. Any nonzero element of $S$ generates a subalgebra of dimension $\geq 2$. Otherwise $x=\alpha e$ for an idempotent $e$ with norm 1 and $\alpha \in F$; but then $n(x)^{3}-\left(x, x^{2}\right)^{2}=\alpha^{6}-\alpha^{6}=0$ which is not possible.

Lemma 6. For any $x \in S$ the subspace $\operatorname{span}\left(x, x^{2}, x^{3}\right)$ is commutative.

Proof. By (13) we have $\left[x^{2}, x^{3}\right]=0$; the linearization of (11) gives $\left[x, x^{3}\right]=0$.

Lemma 7. If $\operatorname{alg}(x\rangle=\operatorname{span}\left\langle x, x^{2}, x^{3}\right\rangle, \forall x \in S$, then $A$ has degree 2 .

Proof. We will proceed by contradiction. By density we can assume that there exists $x \in S$ with $\operatorname{alg}(x) \neq \operatorname{span}\left\langle x, x^{2}\right\rangle$. Then, by hypothesis and the previous note, 
$\operatorname{dim} \operatorname{alg}\langle x\rangle=3$. This means that $\operatorname{alg}(x)$ is not a composition algebra (recall the possible dimensions of composition algebras are $1,2,4,8$ or $\infty$ ). Thus, there must exist $0 \neq z \in \operatorname{alg}\langle x\rangle$ such that $(z, \operatorname{alg}(x))=0$ and the set $\left\{x, n(x) x^{2}-\left(x, x^{2}\right) x, z\right\}$ becomes an orthogonal basis of $\operatorname{alg}(x)$. Since, by the previous lemma, $\operatorname{alg}(x)$ is commutative, we can use Lemma 4 with $y=n(x) x^{2}-\left(x, x^{2}\right) x$ to obtain that $n(x) y z=0$. But $n(x) \neq 0$, so $y z=0$ and the left multiplication fails to be bijective; that is, $n(y)=n(x)\left[n(x)^{3}-\left(x, x^{2}\right)^{2}\right]=0$, which is a contradiction since $x$ belongs to $S$.

Now everything is ready for us to prove that $A$ has degree 2 . We will denote $\left(x^{2} x\right) x$ by $x^{4}$.

Proposition 3. Any third power associative composition algebra of finite dimension over a field of characteristic $\neq 2$ has degree $t w o$.

Proof. Let us start by linearizing the identity (13),

$$
\begin{gathered}
2(x, y) x^{2} x^{2}+n(x)(x y+y x) x^{2}+n(x) x^{2}(x y+y x)+4(x, y) n(x) x^{2}+n(x)^{2}(x y+y x)= \\
2\left(y, x^{2}\right) x^{3}+2(x, x y+y x) x^{3}+2\left(x, x^{2}\right)\left(y x^{2}+x(x y+y x)\right) .
\end{gathered}
$$

Now, we substitute $y=x^{2}$ in this equation and use Lemma 6 to obtain

$$
\left(x, x^{2}\right) n(x) x^{2}-\left(x, x^{3}\right) x^{3}=\left(x, x^{2}\right) x^{4}-n(x) x^{2} x^{3} .
$$

On the other hand, equation (12) with $y \mapsto x^{3}, x \mapsto x$ and $y \mapsto x^{3}, x \mapsto x^{2}$ gives

$$
\begin{gathered}
n(x) x^{3} x^{3}+n(x)^{3} x^{2}=2\left(x, x^{3}\right) x^{4} \\
n(x)^{2} x^{3} x^{3}+n(x)^{3} x^{2} x^{2}=2\left(x^{2}, x^{3}\right) x^{3} x^{2}
\end{gathered}
$$

If we multiply by $n(x)$ the first of these equalities and then we subtract the second one, we get

$$
n(x)^{4} x^{2}-n(x)^{3} x^{2} x^{2}=2 n(x)\left[\left(x, x^{3}\right) x^{4}-\left(x, x^{2}\right) x^{2} x^{3}\right]
$$

This identity can be simplified by choosing $x \in S$, dividing by $2 n(x)$ and using (13):

$$
n(x)^{3} x^{2}-n(x)\left(x, x^{2}\right) x^{3}=\left(x, x^{3}\right) x^{4}-\left(x, x^{2}\right) x^{2} x^{3}
$$

As usual, by density this relation holds for any $x \in A$.

Now, let us consider the set $T=\left\{x \in S \mid\left(x, x^{2}\right)^{2}-n(x)\left(x, x^{3}\right) \neq 0\right\}$. If $T$ were empty then we would multiply (14) by $\left(x, x^{2}\right),(15)$ by $n(x)$ and we would subtract to obtain

$$
n(x)\left[\left(x, x^{2}\right)^{2}-n(x)^{3}\right] x^{2}=\left(x, x^{2}\right)\left[\left(x, x^{3}\right)-n(x)^{2}\right] x^{3} \quad \forall x \in S .
$$


Simplifying by $x$, we conclude that $x$ and $x^{2}$ are linearly dependent, which is not possible as the note below Lemma 5 shows. Hence, $T$ is a dense nonempty open subset. Now, we can look at equations (14) and (15) just as a system of two equations where the indeterminates are $x^{2} x^{3}$ and $x^{4}$, and we would like to solve it in terms of $x^{2}$ and $x^{3}$. This solution will exist if the determinant of the matrix of the coefficients is nonzero, that is, $n(x)\left(x, x^{3}\right)-\left(x, x^{2}\right)^{2} \neq 0$; but this is precisely what is verified by any element of $T$, so for any $x \in T$ we obtain that $x^{4}, x^{2} x^{3} \in \operatorname{span}\left(x, x^{2}, x^{3}\right)$. Moreover, by (12) we can ensure that $x^{3} x^{3}$ and $x^{2} x^{2}$ also lie in this subspace, so it is closed under products; in other words, $\operatorname{alg}\langle x\rangle=\operatorname{span}\left(x, x^{2}, x^{3}\right\rangle \forall x \in T$. By density this holds for any $x$ in $A$. Now the proposition follows from Lemma 7.

Theorem 5 follows from this proposition, Proposition 2, and Theorems 2 and 4.

\section{REFERENCES}

1. J. A. CuEnCA, On one-sided division infinite-dimensional normal real algebras, Publ. Mat. 36 (1992), 485-488.

2. J. A. Cuenca, A. Elduque and J. M. Pérez-Izquierdo, Power associative composition algebras, to appear.

3. A. ElduQue, Symmetric composition algebras, J. Algebra 196 (1997), 283-300.

4. A. Elduque, Okubo algebras and twisted polynomials, Contemp. Math. 224 (1999), 101-109.

5. A. Elduque and H. C. Myung, On Okubo algebras, in Forty Years of Rochester Symposium: From Symmetries to Strings (A. Das, ed., World Scientific Publ., River Edge, New Jersey, 1990), 299-310.

6. A. Elduque and H. C. MYUNG, Flexible composition algebras and Okubo algebras, Comm. Algebra 19 (1991), 1197-1227.

7. A. Elduque and H. C. Myung, On flexible composition algebras, Comm. Algebra 21 (1993), 2481-2505.

8. A. Elduque and J. M. Pérez-Izquierdo, Third power associative composition algebras, Manuscripta Math. 84 (1994), 73-87.

9. A. Elduque and J. M. Pérez-Izquierdo, Composition algebras with associative bilinear form, Comm. Algebra 24 (1996), 1091-1116.

10. A. Elduque and J. M. Pérez-Izquierdo, Composition algebras with large derivation algebras, J. Algebra 190 (1997), 372-404.

11. A. Elduque and J. M. Pérez-IzQuierdo, Infinite dimensional quadratic forms admitting composition, Proc. Amer. Math. Soc. 125 (1997), 2207-2216.

12. K. El-Amin, $M^{\wedge}$ I. Ramírez and A. Rodriguez Palacios, Absolute-valued algebraic algebras are finite dimensional, J. Algebra 195 (1997), 295-307.

13. N. JaCoBson, Composition algebras and their automorphisms, Rend. Circ. Mat. Palermo (2) 7 (1958), 55-80.

14. I. KaPlansky, Infinite-dimensional quadratic forms admitting composition, Proc. Amer. Math. Soc. 4 (1953), 956-960. 
15. M. A. Knus, A. S. Merkurjev, M. Rost and J. P. Tignol, The Book of Involutions, (Amer. Math. Soc. Colloquium Publ., vol. 44, Providence, 1998).

16. H. C. MyUNG, Non Unital Composition Algebras (Global Analysis Research Center, Lecture Notes Series N. 22, Seoul, 1994).

17. S. OKuвo, Pseudo-quaternion and pseudo-octonion algebras, Hadronic J. 1 (1978), $1250-1278$.

18. S. OKuво, Dimension and classification of general composition algebras, Hadronic J. 4 (1981), 216-273.

19. S. OKuвo, Classification of flexible composition algebras, I and II, Hadronic J. 5 (1982), 1564-1626.

20. S. OKuBo and J. M. OsBorn, Algebras with nondegenerate associative symmetric bilinear form permitting composition, Comm. Algebra 9 (1981), 1233-1261.

21. S. OKuBo and J. M. OsBorn, Algebras with nondegenerate associative symmetric bilinear form permitting composition II, Comm. Algebra 9 (1981), $2015-2073$.

22. H. Petersson, Quasi-composition algebras, Math. Sem. Univ. Hamburg 35 (1971), 215-222.

23. A. Rodriguez Palacios, One-sided division absolute valued algebras, Publ. Mat. 36 (1992), 925-954.

24. A. Rodríguez Palacios, Absolute valued algebras of degree two, in Non-Associative Algebra and Its Applications (S. González, ed., Kluwer Academic Publ., 1994), 350-356.

25. K. Urbanik and F. B. Wright, Absolute valued algebras, Proc. Amer. Math. Soc. 11 (1960), 861-866.

26. K. A. Zhevlakov, A. M. Slin'ko, I. P. Shestakov and A. I. Shirshov, Rings that are Nearly Associative (Academic Press. New York, 1982).

Departamento de Matemáticas

Facultad de Ciencias

UNIVERSIDAD DE ZARAGOZA

50009 ZARAGOZA

SPAIN
Department of Mathematics

UNIVERSITY OF WISCONSIN

MADISON, WI 53706

U.S.A.

Present address

Departamento de Matemáticas y Computacioñ

UNIVERSIDAD DE LA RIOJA

26004 LOGROÑO

SPAIN 